Ana Leonar Pereira João Rui Pita

Pedro Ricarda Fonseca (eds.)
Darwin,

Evalution,

Evolutionisms

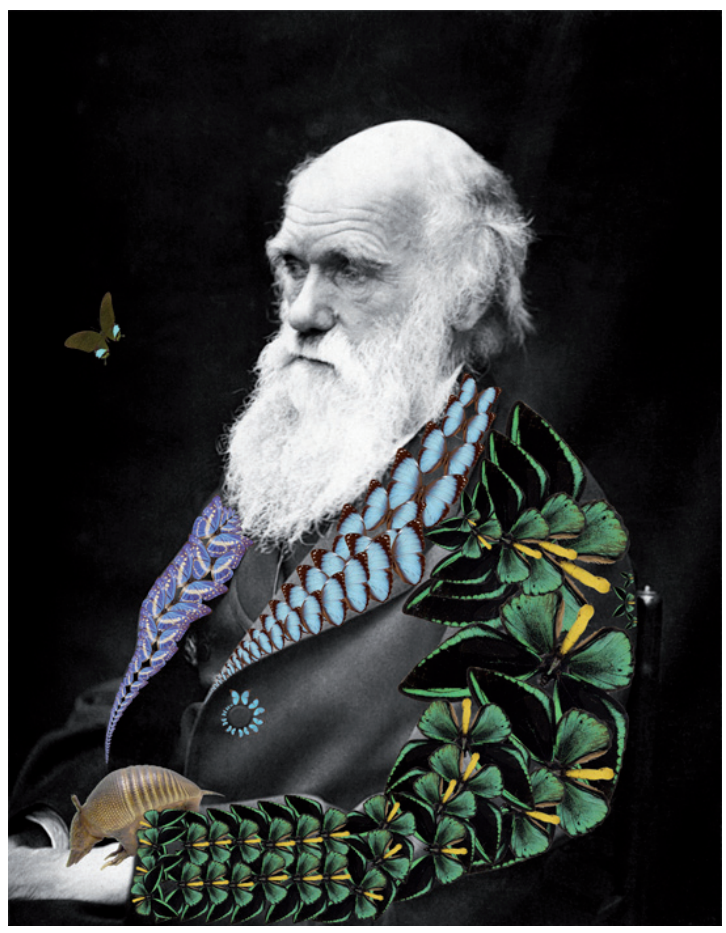


Gökhan Akbay

Department of Philosophy; Middle East Technical University, Ankara, Turkey

\section{Function, Natural Selection and Information}

Functions debate was at first a debate about teleology. The question was whether biological entities were designed by an omnipotent God or the outcomes of a natural process. Now, an omnipotent God has lost its significance as an alternative to naturalistic theories of evolution. However, the apparent harmony and complexity of the biological world still charms many scientists, including arch-evolutionists like Richard Dawkins. ${ }^{1}$ Functions debate turned out to be a debate internal to naturalism.

The vital question in functions debate, namely the substantive question, involves the nature of functional entities. I will primarily be concerned with this question which is about the nature of functional entities. They include natural entities like enzymes, organs, systems, etc. They also include artifacts that humans produce and use. The question is whether we can find an interesting property that is common to all and will unite them except nonfunctional entities.

What do we mean when we say that the function of mirrors is to reflect light? And what does a biologist mean when he says "the function of hemoglobin is to carry oxygen to tissues"? For mirrors we can say they are very good at reflecting light and we use them to reflect light. We can also appeal to the design intentions of mirror producers. In the second case, we can neither find designers nor users of hemoglobin molecules. Natural functions pose more serious problems than conscious functions. In order to justify our usage of the word 'function' we should find a covering definition for both types (conscious and natural).

The most important distinction for Wright is between function and accident. ${ }^{2}$ If we turn back to the hemoglobin example, it is a matter of fact that hemoglobin can also bind to $\mathrm{CO}$ (carbon monoxide), which is a lethal toxin but is not the function of hemoglobin it is rather an accidental side-effect of hemoglobin's structure. The same can be said for artifacts. The computer has many functions but I would think twice before saying "the function of computers is to make noise". This distinction is the central theme of Wright's article.

After a lengthy discussion of previous analyses of function statements, Wright proposes his own solution to the problem. For Wright, the most critical point of

${ }^{1}$ Dawkins,R., "Universal Darwinism”, in Hull, D. and Ruse, M. (eds.), The Philosophy of Biology (Oxford: Oxford University Press, 1998)

${ }^{2}$ Ibid. 
function ascriptions is to explain the reasons for the presence of functional entities ${ }^{3}$. For example, when we claim that the function of knives is to cut, we mean that knives are present because they cut. In the case of artifacts, the point is usually clear. An artifact may have many effects, but one effect can explain why it is there. We can use CDs for ornamenting our cars but they are produced for storing information. Wright asserts that the same point applies to biological functions as well. Hemoglobin is present because it carries oxygen. It may have other effects such as binding to $\mathrm{CO}$, but natural selection has maintained and spread hemoglobin for its oxygen binding capacity.

The second substantial contribution to functions debate comes from Robert Cummins' 1975 article. The article was an attempt to explicate the real aim of functional explanations in science. According to Cummins, functional explanations have nothing to do with the presence of some entity. In contrast, functional explanations seek to explain what contribution an entity or a process makes to the capacities of a containing system. Function attributions thus explain how a containing system produces complex outcomes by means of simple processes and entities.

Cummins claims that the apparent scientific plausibility of selected-effects approaches originates from a misunderstanding of evolutionary theory. According to Cummins, selected-effects approaches falsely consider natural selection as an explanation of the presence of functional traits of organisms. For Cummins, natural selection does not determine which traits an individual has. It is determined by the genetic plan. Natural selection just explains why some traits are more frequent than others by referring to their adaptive advantages. In his own words "natural selection cannot alter a plan, but it can trim the set." ${ }^{4}$

Flowchart diagrams, abstract descriptions of electronic circuits or assembly lines are best examples of Cummins' approach. Consider the computer fan example I mentioned before. The function of the fan is to cool the processor. Cooling the processor is a capacity of the computer which is achieved by the inner workings of the fan. The fan can be decomposed into its simpler parts. These simpler parts contribute to the cooling capacity of the fan, hence, one can explain how the cooling capacity of the computer is realized by means of analyzing relevant parts into simpler and simpler capacities they have.

Wright's line of inquiry was elaborated by Ruth Millikan in her theory of "proper functions". 5 She constructed a theoretical definition that would unite purposeful phenomena under one definition.

Millikan adds a further constraint on functional entities: reproduction. By reproduction she means something like copying. Copying occurs when some properties of an original entity determine the properties of its descendants in a regular way. For instance, the copying machine maps the written marks of the original paper onto another sheet of paper and produces a similar paper. In copying, descendants systematically co-vary with the original entity (the ancestor or the model).

\footnotetext{
3 Ibid. p. 154

${ }^{4}$ Ibid. p.751

${ }^{5}$ Millikan, R. Excerpt from Language, Thought and Other Biological Categories in David Buller (Ed.), Function, selection, and design, Albany, N.Y. : State University of New York Press, 1999.
} 
Another constraint Millikan adds is selection. For Millikan, we cannot determine the function of a trait just by looking at its present benefits. The point is that we cannot distinguish fortuitous benefit from genuine function by just looking at its present performance. How beneficial should a trait be in order to be functional? Millikan identifies two extremes regarding biological cases ${ }^{6}$. The first extreme is the view that the trait in question should be a necessary component of the living system. Homeostatic functions (i.e. thermoregulation in haemothermal animals and such necessary dispositions of an organism) may be a candidate for this extreme view. However, even homeostatic functions were not present in some ancestors of today's organisms. So they cannot be "necessary" in the strict sense. There are also some functions of traits that are not performed in the lifetime of an individual. Mating displays of a lonely budgie would not occur unless a friend from opposite sex is placed into his cage.

Another extreme is the view that a past token might have a function just because it has benefited only once in the reproduction of the trait. As Millikan states it, it is natural selection that draws the line between these two extremes. Hence, selection is a necessary part of proper function definitions. In her own words: "Whether something has a function is not a matter of how often it has accidentally helped out in the movement from generation to generation. Anything whatever might occasionally have done that. It is a matter of whether it was selected to help out in this way."7

A crucial point of Millikan's account is the reference to historical conditions. As we observed before, it is the complexity of the system and relevant simplicity of dispositions that make a causal role analysis of functions plausible. It is the explanatory aims of the researchers that determine which capacities would deserve analysis. This may be true for machines in general. They are specified by certain rules of performance. There are rules that specify the proper input, internal states and expected output. This is why abstract characterizations like flowchart diagrams are well suited to describing machines.

The central problem with Cummins' account is that it cannot capture the normative aspect of function ascriptions ${ }^{8}$. For Millikan, since Cummins does not ascribe functions to parts that do not actually contribute to a systemic capacity, his analysis cannot account for malfunctions. According to Millikan, an entity has a proper function not because of its contemporary dispositions. Having a function depends on history. The historical link between normativity and function is provided by natural selection.

Reference to natural selection has been seriously criticized by many philosophers. Counter examples include swampman ${ }^{9}$ (a molecule to molecule duplicate of a person who randomly and immediately appears), screws that accidentally fall into a machine and make a functional connection ${ }^{10}$, brand-new antibiotic resistance genes that enter

${ }^{6}$ Ibid. p. 35

${ }^{7}$ Ibid. p. 38

${ }^{8}$ Millikan, R. (1989) “In Defense of Proper Functions". Philosophy of Science 56: pp. 294-295

9 Sterelny, K. and Griffiths, P. Sex and Death: An Introduction to Philosophy of Biology (Chicago: University of Chicago Press, 1999), p.222

${ }^{10}$ Kitcher, P. "Function and Design," in Hull, D. and Ruse, M. (eds.), The Philosophy of Biology (Oxford: Oxford University Press, 1998) 
a bacterium ${ }^{11}$, etc. All of these attempts aim to show one thing: history is not essential to function ascriptions. These counterexamples seem to miss the point in a crucial respect. If Millikan were doing conceptual analysis, in other words if she were trying to determine the criterion for true usages of the term function these examples would show that including selection and even reproduction in the definition of function would lead to constant failures. However, Millikan explicitly states that she is not in search for a clarification of the older versions of function concept ${ }^{12}$. Rather, she was trying to find a theoretical definition of purposeful phenomena. Although she does not explicitly tell what the difference will be, I can cite one property of theoretical definitions: they may conflict with your intuitions. Consider "burning". A theoretical definition of burning would cite chemical reactions which involve oxidation. Thus, corrosion would be burning according to the theoretical definition. However, someone who lacks that theoretical knowledge would find this characterization counterintuitive. It is not a conceptual necessity that functional things have a selection history. It is how things work on our planet.

Discussions in functions debate looks very much like the intentionality debate. Just consider how many themes coincide in two fields: malfunction-misrepresentation, normativity of proper functions-normativity of content, algorithmicity of natural selection-algorithmicity of thought, etc. the list can be extended. Is the use of such similar concepts just a matter of chance or are there real similarities between two fields? In my opinion similarity is real and it is because of the semantic aspects of these two fields. By semanticity I do not mean lingual meaning. What I mean is a systematic covariance with some aspects of environment that could count as a normative relation.

The greatest problem for both of these fields is to naturalize normativity (or eliminate it). Since there is such an overlap between these fields, I think we can borrow some concepts from one and apply them to the other. I would like to borrow the concept of information from intentionality debate and investigate whether it can give us the unifying definition we want. I have two aims in this part of my essay. The first one is to explain the intuitions that led me to carry out this project. The second is to begin my investigation by finding out what kind of an information concept can give us the unity we want: unity of artifactual and biological domains, unity of proper functions and causal role functions.

I am impressed by the idea that functional structures carry information about their environment ${ }^{13}$. Function is, in a sense, information about the past environments where organisms evolved. All functions are selective. Their domains are specific. For instance, a bird's wings are adapted to fly only in a fluid which has a viscosity similar to the Earth's air. All of the enzymes are highly specific to their substrates. Eyes have a specific range of wavelengths for sight. It seems as if natural selection coded relevant information into our genes. The case is also relevant to artifacts. Artifacts

\footnotetext{
11 Sterelny, K. and Griffiths, P. (1999) p.222

12 Millikan, R. (1989a) p.293

${ }^{13}$ Dennett, D. C., Darwin's Dangerous Idea: evolution and the meanings of life. New York : Simon \& Schuster, 1996. p. 198
} 
are also domain specific. Their specificity depends on the things on which they are used. For example a pen is specific to some kinds of surfaces, you cannot write on anything. In a sense, a pen includes information about its substrate. This is the first intuition that affects me.

The second intuition comes from biological practice. The use of information concepts in biology, especially in genetics is very common. In my opinion, this is no arbitrary choice. Informational concepts fit this area well. It is apparent from the successes of this approach. Geneticists usually talk about coding, instructions and other such concepts. As I mentioned before, the use of flowchart diagrams in molecular biology also supports such an intuition. Flowchart diagrams or more detailed algorithms tell us how under specified conditions a program will respond. If these fit so well in molecular biology, can we conclude that there really is information about those conditions coded into the organisms themselves? Let me clarify this point. In biochemical pathways molecules are depicted as activating or inhibiting each other. For instance we can describe a pathway like this: if there is this much bicoid proteins nearby, activate such and such genes and begin segmentation, else do nothing.

These are just intuitions and need clarification. The first point of clarification regards the concept of information. What kind of an information concept should we have in order to account for these facts? There are two types of information concepts in use. One was first discovered by Claud Shannon. Shannon's information concept measures the nonaccidental correlation between variables. ${ }^{14}$ For instance, smoke gives information about the presence of fire because they show a correlation. Another example may be the bell's ringing. This sense of information is only inferential. ${ }^{15}$ To account for functions, we should find another concept of information. We need it in order to capture normative aspects of functions.

The second type of information is called "semantic information". As Dretske describes it, semantic information depends on nomic regularities between the source and the signal ${ }^{16}$. Semanticity is not identical to meaningfulness. According to Dretske, the difference between information and meaning is that having the information that $\mathrm{P}$ automatically guarantees having any other nomically or analytically nested information. Analytical nesting is logical implication. Nomical nesting is making some state of affairs necessary by means of a law of nature. For example "this ball is red" carries the information that "this ball is not blue" because it implies that logically but it does not mean that.

I claim that this semantic concept of information can give the unified notion of function. First of all, it is normative but it also accords with a synchronic analysis. Secondly, whatever the physical details of coding information are, both design processes and natural selection can code relevant environmental information into artifacts and organisms.

\footnotetext{
${ }^{14}$ Godfrey-Smith, P. "Information in Biology" in Hull, D. and Ruse, M. (Eds.), The Cambridge Companion to Philosophy of Biology, (Newyork: Cambridge University Press, 2007), p.106

${ }^{15}$ Ibid. p. 107

${ }^{16}$ Dretske, F. Knowledge and the flow information, (Cambridge, Mass. : MIT Press, 1981), p.72
} 